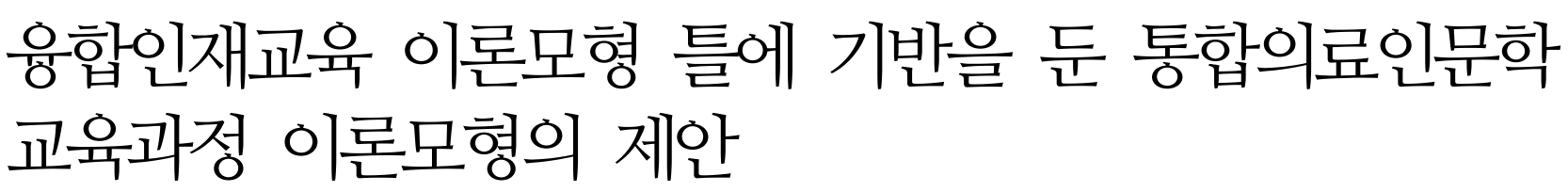

김진희 ${ }^{1} \cdot$ 이영환 ${ }^{2} \cdot$ 박원균 $^{1} \cdot$ 박영순 $^{2} \cdot$ 박혜진 $^{1} \cdot$ 천경희 $^{2}$

${ }^{1}$ 계명대학교 의과대학 의학교육학교실, ${ }^{2}$ 영남대학교 의과대학 의료인문학교실

\title{
The Development of a Theoretical Model of Integrated Medical Humanity Curriculum Using Science, Technology, Engineering, Arts, and Mathematics Model
}

\begin{abstract}
Jin Hee Kim ${ }^{1}$ - Young Hwan Lee ${ }^{2}$ Won Kyun Park ${ }^{1}$ Young Soon Park ${ }^{2} \cdot$ Hae Jin Park ${ }^{1}$ Kyung Hee Chun ${ }^{2}$
${ }^{1}$ Department of Medical Education, Kyemyung University School of Medicine; ${ }^{2}$ Department of Medical Humanities, Yeungnam University College of Medicine, Daegu, Korea
\end{abstract}

\begin{abstract}
The purpose of this study was to propose a theoretical model for an integrated medical humanities curriculum based on a STEAM (science, technology, engineering, arts, and mathematics) education framework and to provide a guideline for curriculum integration. Three dimensions of integrated curriculum development are competencies, core contents, and elements of integration. Competencies imply the purpose of the medical humanities of a medical school and the exit outcomes of the curriculum. Core contents imply the goals and objectives of the curriculum. We compared the goals and themes of the medical humanities with core attributes of professionalism. Four elements of integration were proposed: units (cases, problem activities, core contents, disciplines/subjects), types (multidisciplinary, interdisciplinary, transdisciplinary), contexts of integration (life cycle of patients, scope of society), and stages of student development (from student to doctor). It is expected that this theoretical model for an integrated medical humanities curriculum can be used as a guideline for curriculum development and an evaluation criterion for instructional designers and subject matter experts.
\end{abstract}

Keywords: Medical humanities, Curriculum, Theoretical models

\author{
Corresponding author \\ Kyung Hee Chun \\ Department of Medical Humanities, \\ Yeungnam University College of \\ Medicine, 170 Hyeonchung-ro, \\ Nam-gu, Daegu 705-717, Korea \\ Tel: $+82-53-620-4380$ \\ Fax: + 82-53-628-4383 \\ E-mail: khchun@yu.ac.kr \\ http://orcid.org/0000-0002-5351-0376 \\ Received: Janurary 2, 2015 \\ 1st revised: February 9, 2015 \\ 2nd revised: February 16, 2015 \\ Accepted: February 16, 2015
}

\section{서 론}

의과대학의 교육목적은 미래의 의료 전문가, 즉 좋은 의사의 양성 에 초점 맞추어져 있다. 이때 좋은 의사란 의사로서 우수한 임상능력 과 바른 직업관을 가지고 있고 환자로부터 두터운 신뢰를 받고 있는 사람으로, 이때 바른 직업관이란 높은 윤리성, 도덕성, 깊은 신뢰감, 겸손함은 물론 다른 의료인과의 팀워크, 한계 인식, 평생 공부의 책임감을 가진 사람을 의미한다(Baik, 2010). 오랜 기간에 걸쳐 의학 지식의 전수와 생-의학적 모형에 의한 질병과 치료에 관한 지식을 우선하여 교육해 온 전통으로 인해 이러한 태도와 소양, 즉 정의적 특성들은 의과대학에서의 교육 대상이기보다는 졸업 후 체험을 통한 전수의 대상이 되어 왔다. 한국의과대학. 의학전문대학원협의회는 과거 의과대학의 교육이 인간의 질병에 대한 통합적인 이해와 접근
능력을 가지지 못하고 주로 생물학적 측면에만 편중되어왔으며, 의학 이 분명 인간을 대상으로 하고 있음에도 자연과학이라는 틀에 갇혀서 인간을 하나의 물체로 대하여 온 경향이 있어왔음을 지적한 바 있다 (The Korean Council of Medical College Deans, 2007). Kwon (2005) 또한 그간 의학이 과학적 의학을 지향하면서 환자보다 질병에 관심을 기울여 의학의 인간적인 측면을 등한시 한 바를 의학계 내에 서 문제로 인식하고 이를 보강하여 인간적인 의학(humane medicine) 을 만들고자 하였으며, 이에 따라 의료인문학이 등장하게 되었다고 하였다. 그러나 이러한 인문사회의학 즉 의료인문학1)은 새롭게 만들 어진 것이 아니라 의료의 본질과 의료행위에 대한 오랜 철학적 숙고

\footnotetext{
${ }^{1)}$ 인문사회의학, 의료인문학, 의인문학 등 다양한 용어가 사용되고 있으나, 본 연구에서는 이러한 영역들을 총칭하여 의료인문학으로 통일하여 기술함.
} 
가 보다 명시적이고 체계적으로 드러나게 된 것이자 의학교육 내부의 자기 반성적 성찰의 결과로 재조명되고 있는 것이라 할 수 있다.

Jeon et al. (2010)이 쓴 '인문사회의학'에서도 의료인문학 교육의 필요성은 잘 드러난다. 저자들은 첫째, 의사는 자기 자신을 도구로 사용해서 인간을 직접 다루는 직업에 종사하며, 둘째, 의사는 인간의 총체적 고통 앞에 서 있는 사람이고, 셋째, 의사는 팀으로 일하는 직업이며, 넷째, 의사는 가치에 대한 의사결정을 하는 직업이고, 다섯째, 의사는 사회가 제공하는 자원분배시스템 속에서 일하는 존 재이고, 마지막으로 의사는 내적 성찰을 해야 하는 존재이기 때문에 의료인문학 교육이 필요하다고 말하고 있다. 이러한 의료인문학 교 육의 필요성, 즉 '왜?'라는 질문은 무엇을, 어떻게 교육하고 평가할 것인가에 대한 질문들로 이어져 왔다. 따라서 의료인문학 교육과정 은 고등학교를 졸업한 우수인재인 우리의 학생들이 점차 좋은 의사 로 성장해 가고 사회화되어 가는 일련의 '의사되기(doctoring)' 과정 이어야 하며, 좋은 사회 시민으로의 성장을 돕기 위한 교양교육에서 시작하여 좋은 의사가 갖추어야 할 역량과 직업전문성을 갖출 수 있도록 교육내용과 학습활동을 구성해야 한다.

의료인문학 교육과정의 필요성, 내용, 방법과 평가 등에 대한 해답을 찾는 과정은 지금도 여러 의과대학의 교육과정 개선작업과 연구들을 통해 이루어지고 있다. 특히 1 개 의과대학의 의료인문학 교육과정 개선에 대해 기술한 Jeon \& Yang (2003)의 저서와 의료인 문학 교육과 관련한 국내외 정보와 제안을 제공해주는 Jeon et al. (2010)의 저서는 의료인문학 교육의 내용, 방법, 평가에 대한 이해에 도움이 된다. 그러나 다수 의과대학에서 의료인문학 교육과정이 개 발되고, 그 교육방법에 대한 다양한 시도가 이미 이루어지고 있음에 도 불구하고 개별교과가 아닌 통합 교육과정으로서의 의료인문학 교육을 위한 교육과정 개발 가이드라인이 명확하게 제시되지 못했 다. 따라서 본 연구진은 의과대학 현장에서 의료인문학 관련 교육과 정 개발자들과 운영자들이 의료인문학 통합 교육과정을 개발하고 실행하는데 있어 도움이 될 수 있는 이론모형, 교육과정 구성과 관련한 가이드라인이 될 표준의 개발이 요구된다는데 의견을 같이하 고 의료인문학 교육과정의 총체적 방향과 통합적 접근을 위한 이론 모형에 대하여 고찰해 보기로 하였다.

특히 Kim et al. (2012)이 제안한 융합인재교육(science, technology, engineering, arts, and mathematics, STEAM 교육)의 이 론적 모형 틀에 준하여 통합의료인문학 교육과정을 위한 이론 모형을 도출하고자 하였으며, 그 과정은 형성적 연구(formative research) 기법(Reigeluth, 1999)을 활용하여 이루어졌다. 즉 의료인문학 통합 교육의 다양한 요소들을 검토하고, 각 요소들과 관련된 해당 사례들 을 수집하여 모형 적용 가능성을 탐색한 후, 최종 수정작업을 거쳐 잠정적 이론모형을 제안하고 각 구성요소들에 대해 설명하고자 한다.

\section{교육과정 통합의 유형과 이론 틀로서의 STEAM 모형}

본 연구에서는 이론모형을 도출하기 위한 기본 틀로서 EwhaSTEAM 융합모형(Kim et al., 2012)을 사용하였다. 이 모형은 지식, 역량, 그리고 통합 방법을 이론적 차원으로 해석하고 설명하고자 하 였으며, 교육과정 통합의 대표적 접근방법들을 융합 요소 차원에서 충분히 고려하고 적용할 수 있다. 교육과정 통합을 위한 대표적 접근 방법으로는 Wolfinger \& Stockard (1997)가 제안한 학문분야 중심 의 통합, 언어 및 문학 중심의 통합, 주제 중심의 통합, 그리고 쟁점 중심의 통합을 들 수 있다. Kang \& Ju (2004)는 학문분야 중심의 통합이란 서로 구별되는 둘 이상의 지식체계를 통합하는 것으로서 지식의 여러 범주를 묶는 방식이라 하였으며, 언어와 문학중심 통합 은 학교 교과 내의 통합과 교과 간의 통합을 위한 도구로 문학작품을 활용하는 방법이라 하였다. 또한 주제중심 통합은 핵심 주제를 중심 으로 탐구와 학습이 이루어지도록 하는 방식이며, 쟁점 중심 통합은 사회 쟁점과 문제를 조사하여 통합하는 방식으로 학생들에게 유의 미한 강한 동기를 부여할 기회를 제공할 수 있다고 하였다.

STEAM 교육은 2011년 한국의 초중등교육 강화를 위해 한국 정부에서 도입한 교육정책의 하나다. 2005년부터 미국 Obama 정부 가 추진한 초기 STEM 교육은 과학(science), 기술(technology), 공 학(engineering), 그리고 수학(mathematics)을 통합적으로 교육하 는 것을 의미하는데, 이를 보다 확장한 STEAM 모형은 과학, 기술, 공학, 수학뿐만 아니라 예술(arts)을 포함시켰으며, 궁극적으로 분석 적 사고와 창의적 사고를 가진 학생들에 대한 사회적 요구가 반영된 통합교육으로의 접근이다. 특히 Baek et al. (2012)은 미래의 인재는 새로운 발상과 도전으로 창의성을 발휘하고, 문화적 소양과 다원적 가치에 대한 이해를 갖추며, 세계와 소통하는 시민으로서 배려와 나눔의 정신으로 공동체 발전에 기여할 수 있어야 하며, 이러한 급변하는 사회적 요구를 반영하여 창의와 인성을 지닌 미래 융합형 인재를 양성하는 것을 융합인재교육의 방향이라 밝힌 바 있다. Kim et al. (2012)은 융합인재교육을 위한 이론적 모형을 제안하기 위한 연구에서 창의융합형 인재의 능력 및 자질은 무엇인지, 서로 다른 학문간 융합이 가능한지와 가능하다면 어떻게 가능한지, 그리고 방 법론적으로 융합인재교육을 어떻게 실현할 것인지를 묻는 3 가지 질문을 하였고, 이에 따라 핵심지식, 핵심역량, 융합요소의 3개 차원 으로 이론적 모형을 설명한 바 있다(Table 1).

Ehwa-STEAM 융합인재모형은 비록 초·중등 교육 실무자를 위한 가이드라인을 제공하기 위한 목적으로 개발되긴 하였으나, 상 이한 학문영역의 통합을 위하여 관련 영역의 핵심 지식과 그 결과로 서의 역량, 그리고 다양한 통합요소2)들을 규명하고 통합요소들에

\footnotetext{
${ }^{2)} \mathrm{Kim}$ et al. (2012)의 연구에서는 융합(convergence)으로 명명하였으나 본 연구에서는 의학교육에서 주로 사용되고 있는 통합(integration)으로 통칭한다.
} 
Table 1. Three dimensions of the Ewha-STEAM education model ${ }^{a}$

\begin{tabular}{lcc}
\hline \multicolumn{1}{c}{ Key knowledge } & Key competency & Elements of convergence \\
\hline Textbook based core idea & Textbook-based integration competency & Unit of convergence \\
Patterns & Asking questions \& defining problems & Concept/inquiry process \\
Cause and effect & Developing \& using models & Problem/phenomenon \\
Scale, proportion \& quality & Etc. & Activity for experience \\
Systems \& system models & Creativity \& character & Degree of convergence \\
Energy \& matter & Divergent thinking & Multi-disciplinary \\
Structure and function & Imagination/visualization $\cdots$ & Inter-disciplinary \\
Stability \& change & Ecological worldview & Extra-disciplinary \\
Liberal arts & Socioscientific accountability & Context of convergence \\
& & Personal \\
& & Societal \\
\end{tabular}

${ }^{a)}$ Kim et al., 2012.

\begin{tabular}{|c|c|c|}
\hline $\begin{array}{l}\text { Goals of education } \\
\text { (competence) }\end{array}$ & $\begin{array}{c}\text { Core contents } \\
\text { (objectives) }\end{array}$ & Elements of integration \\
\hline $\begin{array}{l}\quad \text { (Examples) } \\
\text { Keywords of competence } \\
\text { - Professionalism } \\
\text { - Self-development } \\
\text { - Communication } \\
\text { - Ethics \& moral reasoning } \\
\text { - Problem solving } \\
\text { - Social \& community-based } \\
\text { health care } \\
\text { - Patient-centered care }\end{array}$ & $\begin{array}{l}\text { - Understanding humanity } \\
\text { \& suffering } \\
\text { - Understanding ethics \& } \\
\text { medical ethics } \\
\text { - Analyzing \& developing } \\
\text { oneself } \\
\text { - Understanding } \\
\text { relationship between } \\
\text { medicine \& society } \\
\text { - Maintaining relationship } \\
\text { with others } \\
\text { - Understanding } \\
\text { professional conduct }\end{array}$ & $\begin{array}{l}\text {-Unit of integration } \\
\text { - Cases } \\
\text { - Problems } \\
\text { - Activities } \\
\text { - Core contents } \\
\text { - Disciplines/subjects } \\
\text { - Type of integration } \\
\text { - Multidisciplinary } \\
\text { - Interdisciplinary } \\
\text { - Transdisciplinary } \\
\text { - Contexts of integration } \\
\text { - Life cycle of patients } \\
\text { - Scope of society } \\
\text { - Level of development }\end{array}$ \\
\hline
\end{tabular}

Figure 1. Three dimensions of theoretical model of integrated medical humanities curriculum.

따른 실천적 방안을 제시한 최신 이론모형이다. 특히 현재 의학교육 에서 주로 추구하는 역량중심 교육과정 혹은 성과바탕 교육과정의 주요 개념을 반영할 수 있다는 점과 통합을 위해 고려해야 할 요소들 을 명확히 제시할 수 있다는 점에서 장점이 있을 것으로 판단되어 이론적 틀로써 적용되었다. Ewha-STEAM 융합모형의 세 차원은 핵심지식(key knowledge), 핵심역량(key competency), 융합요소 (elements of convergence)로 나누어진다. 이때 핵심지식은 융합인 재교육의 목표인 창의·융합형 인재가 갖추어야 할 기본 지식이며, 이는 다시 여러 학문영역에 걸쳐 적용될 수 있는 개념인 교과기반 통합개념(core ideas)과 서로 다른 학문영역을 보다 본질적으로 융합 하기 위한 소양지식으로 구성된다. 창의·융합형 인재가 갖추어야 할 핵심역량은 크게 교과기반 통합역량과 창의· 인성역량으로 나뉘 는데, 이때 교과기반 통합역량은 각 교과를 기본으로 하되, 여러
학문영역에 전이시켜 문제를 해결할 수 있는 역량을 의미한다. 마지 막으로 융합요소 차원은 실제 융합인재교육을 위한 접근방식으로 융합 단위, 융합 방식, 융합 맥락으로 구분되며 이들 요소들을 고려하 여 교육프로그램과 교육과정을 개발 및 실행할 수 있다고 제안하고 있다.

본 연구에서는 3 회에 걸친 숙의과정을 통해 3 개 영역의 1 차 이론 모형을 도출하였다. 이들 모형의 구성요소들에 대한 3명의 의학 전문가와 3 명의 교육학 전문가의 합의적 수정- 개선 작업을 거쳐 1 개의 요소를 추가 구성한 잠정적 이론모형인 통합 의료인문학 교육 과정 이론모형(Theoretical Model of Integrated Medical Humanities Curriculum, IMHC 모형)을 개발하였다. 


\section{통합 의료인문학 교육과정 이론모형: IMHC 모형}

Kim et al. (2012)의 연구모형에 기반을 두어 IMHC 모형을 도출 하기 위한 첫 번째 검토사항은 의료인문학 교육과정이 추구해야 하는 인재상이 무엇인가에 관한 것이었으며, 이후 이러한 인재들이 갖추어야 할 의료인문학적 핵심역량은 무엇인지, 그리고 이러한 역 량을 길러주기 위한 교육내용과 방법은 무엇인지에 대해 논의하였 다. STEAM 이론모형을 기반으로 하여 의료인문학 교육과정 개발을 위해 고려해야 하는 3 개 영역을 도출하였으며, 이는 대학의 교육목표 나 핵심역량, 핵심내용, 그리고 통합요소였다(Figure 1). 또한 이들 영역 외에 고려해야 할 요인으로 학생들의 발달수준을 포함하였다.

\section{1. 핵심역량}

STEAM 모형에서는 교과영역이 과학, 기술, 공학, 예술, 수학으 로 명료화되어 있어 이들 각 교과영역의 교육내용으로서의 지식과 이를 뒷받침해줄 수 있는 소양지식이 가장 먼저 제시되었으나 의료
인문학의 경우 다양한 관련 학문영역이 포함될 가능성을 고려하여 대학의 인재상을 기반으로 한 핵심역량을 먼저 검토할 필요성이 있다. 특히 각 대학 수준의 의료인문학 교육과정 개발에서 가장 먼저 고려해야 할 사항이 바로 해당 대학의 인재상과 그것을 기반으 로 한 졸업역량이다. 본 연구에서는 국외 성과중심교육과정 개발에 관한 $\mathrm{Han}$ (2013)의 연구에서 제시되었던 미국, 영국, 오스트레일리 아의 의과대학 성과(outcome)와 국내 5개 대학의 졸업역량을 검토 해 보았다. 본 연구에서 검토한 것은 스코틀랜드 학장단 내 의학교육 과정 그룹(Scottish Deans' Medical Curriculum Group), Shefield 의과대학, Brown 의과대학, Indiana 의과대학, New South Wales 의과대학, 부산대학교 의학전문대학원, 충남대학교 의과대학/의학 전문대학원, 인제대학교 의과대학, 계명대학교 의과대학, 영남대학 교 의과대학/의학전문대학원의 졸업역량이었다. 국내 의과대학은 최근 교육과정 개선을 시도한 대학들 중 개발된 졸업역량을 홈페이 지, 학회 및 워크숍자료, 학술지 논문 등에서 구체적으로 명시하였던 5 개 대학으로 한정하였다. 10 개 기관의 졸업역량들 중 의료인문학

Table 2. Key words of competencies of 10 medical schools

\begin{tabular}{|c|c|c|}
\hline Index of competency & Frequency & Examples of competency \\
\hline Professionalism & 14 & $\begin{array}{l}\text { Professionalism, professional behavior, professional leadership, leadership, role recognition, dedication, } \\
\text { accountability, responsibility, social responsibility as a doctor, role of the doctor within the health service }\end{array}$ \\
\hline Self-development & 13 & $\begin{array}{l}\text { Self-awareness, self-care, personal growth, personal development, self-development, self-directed learning, } \\
\text { lifelong learning, self-regulated learning, self-management, reflection, reflective practitioner }\end{array}$ \\
\hline Communication & 11 & Communication, effective communication, interpersonal skills, team working, collaboration \\
\hline Ethics \& moral reasoning & 6 & Ethics, moral reasoning, medical ethics, regal responsibility, ethical understanding, ethical judgment \\
\hline Problem solving & 6 & Problem solving, decision making, research, critical thinking \\
\hline $\begin{array}{l}\text { Social \& community-based } \\
\text { health care }\end{array}$ & 6 & $\begin{array}{l}\text { Social responsibility as a doctor, role of the doctor within the health service, social \& community contexts } \\
\text { of health care, social and cultural aspects of health \& disease }\end{array}$ \\
\hline Patient-centered care & 2 & Patient-centered care \\
\hline
\end{tabular}

Table 3. Educational objectives in the development of core contents

\begin{tabular}{|c|c|c|}
\hline & Modified educational objectives of the medical humanities ${ }^{\text {al }}$ & Professionalism ${ }^{\mathrm{bl}}$ \\
\hline $\begin{array}{l}\text { Understanding humanity } \\
\text { \& suffering }\end{array}$ & Understanding humanity, understanding the nature of suffering & Caring \& compassion, respect for the healing function \\
\hline \multirow{2}{*}{$\begin{array}{l}\text { Understanding ethics and } \\
\text { medical ethics }\end{array}$} & Understanding ethics & Integrity \& honesty, morality \& ethical conduct, trustworthiness \\
\hline & Understanding medical ethics & Confidentiality, altruism, trustworthiness \\
\hline \multirow{2}{*}{$\begin{array}{l}\text { Analyzing \& developing } \\
\text { oneself }\end{array}$} & Objective analysis of self & Insight: self-awareness, self-regulation \\
\hline & Continuous self-improvement \& management & Competence, self-regulation \\
\hline $\begin{array}{l}\text { Understanding } \\
\text { relationship between } \\
\text { medicine \& society }\end{array}$ & $\begin{array}{l}\text { Understanding humanities, understanding religion \& culture, } \\
\text { understanding social science, understanding health care, } \\
\text { understanding related fields }\end{array}$ & \\
\hline \multirow{3}{*}{$\begin{array}{l}\text { Maintaining relationship } \\
\text { with others }\end{array}$} & Communication skill & Openness \\
\hline & Relationship with patients & $\begin{array}{l}\text { Respect for patient dignity and autonomy, altruism, } \\
\text { trustworthiness }\end{array}$ \\
\hline & Relationship with colleagues & Trustworthiness, teamwork \\
\hline \multirow[t]{3}{*}{$\begin{array}{l}\text { Understanding } \\
\text { professional conduct }\end{array}$} & Accountability as a doctor & $\begin{array}{l}\text { Presence, commitment, autonomy, altruism, integrity \& honesty, } \\
\text { morality \& ethical conduct, responsibility to the profession }\end{array}$ \\
\hline & Social responsibility of doctor & Altruism, morality \& ethical conduct, responsibility to society \\
\hline & Leadership of doctor & $\begin{array}{l}\text { Autonomy, integrity \& honesty, responsibility to the profession, } \\
\text { responsibility to society, teamwork }\end{array}$ \\
\hline
\end{tabular}

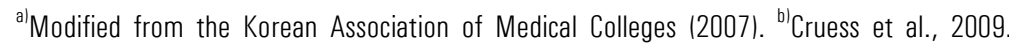


관련 역량을 유목화해 본 결과, 가장 빈번하게 다루어진 역량 키워드 는 프로페셔널리즘, 자기개발, 의사소통, 윤리, 문제해결, 사회와 공동체 기반의 의료, 환자중심 순으로 나타났다(Table 2).

\section{2. 핵심내용}

의료인문학 교육과정 개발에 있어 다음으로 고려해야 할 것은 교육해야 하는 주요 핵심내용들을 확인하는 것이다. 본 연구진은 역량 유목화 과정에서 의료인문학 교육과정이 의사되기(doctoring) 과정으로 해석될 경우 프로페셔널리즘이 매우 강조될 것이라는데 합의하였다. 이에 2007년 한국의과대학. 의학전문대학원협회에서 제시한 의료인문학 교육을 위한 학습목표 분류항목과 함께 Cruess et al. (2009)이 제시한 프로페셔널리즘의 핵심요소들을 함께 비교하 고, 핵심내용의 영역에 대한 숙의과정을 거쳐 Table 3과 같이 핵심 교육내용을 재구성하였다.

핵심 교육내용의 기본 틀은 기존 교육목표영역(The Korean Council of Medical College Deans, 2007)을 사용하였으나 숙의과 정에서 다음과 같은 수정사항들이 도출되었다. 먼저 '의료와 사회의 관계에 대한 이해' 영역은 의과대학 진학 후 교양교육과정에서부터 교육해야 하는 기초영역이자 고학년으로 가면서 점차 심화되어 교육 되어야 할 영역이다. 해당 영역의 하위 학습목표 중, '정치, 경제, 사회에 대한 이해'는 해당 학문 외에도 심리학과 사회복지학 등 사회과학 전반에 대한 교육이 함께 이루어질 필요가 있다는 점에서 ‘사회과학에 대한 이해’로 수정하고, 이러한 이해의 기본이 인문학이 라는 점에 근거하여 '인문학에 대한 이해'를 그 하위영역에 포함하였 다. 그리고 의학 전 교육, 즉 의예과 교육과정에서부터 교육해야 하는 내용의 위계는 '인문학의 이해,' '종교와 문화에 대한 이해,'
'사회과학에 대한 이해,' ‘의학에 대한 이해,' 그리고 '인접학문에 대한 이해'로 구성하는 것에 합의하였다. 이는 프로페셔널리즘과의 비교에서도 알 수 있으며, 해당 내용들이 의료실무와는 원전이 영역 으로 인식되며 자유교과에 해당되는 기본적인 의료인문학적 소양을 의미한다는 점에 근거한 것이다.

다음 수정사항은 직업전문성에 관한 것으로 의료인문학 교육목 표영역의 대부분이 의사의 직업전문성 내용을 내포하고 있으므로 기존 교육목표영역 중 '의사의 직업전문성(professionalism as a doctor)' 영역을 '전문가로서의 품행 이해(understanding professional conducts)'로 수정하고, 그 하위영역을 '의사의 직업적 책 무, '의사의 사회적 책무, '의사의 리더십'으로 정의하였다. 또한 '의료와 사회의 관계에 대한 이해'의 하위영역에서 '의학에 대한 이해(understanding medicine)'를 '의료에 대한 이해(understanding health care)'로 수정하였다.

\section{3. 통합요소}

의료인문학 교육과정 개발에 있어 교육의 성과로서의 역량과 핵 심내용이 규정된 이후 가장 중요하게 고려해야 할 사항은 바로 통합 의 요소들이다. STEAM 이론모형에서는 융합 단위, 융합 방식, 융합 맥락의 세 가지 접근방식을 제안한 바 있다. 본 연구에서도 고려해야 할 3개의 접근방식에 대해 합의하고, 의학교육과 의료인문학 영역의 특성들을 기반으로 하여 통합의 단위(integrating unit), 통합의 유형 (integrating type), 그리고 통합의 맥락(integrating contexts)을 추 출하였다. 또한 통합한 내용이나 교육과정의 교육기간을 산정하기 위한 적정 학생발달수준도 함께 고려할 요소로 도출하였다. 그 도식 은 Figure 2와 같다.

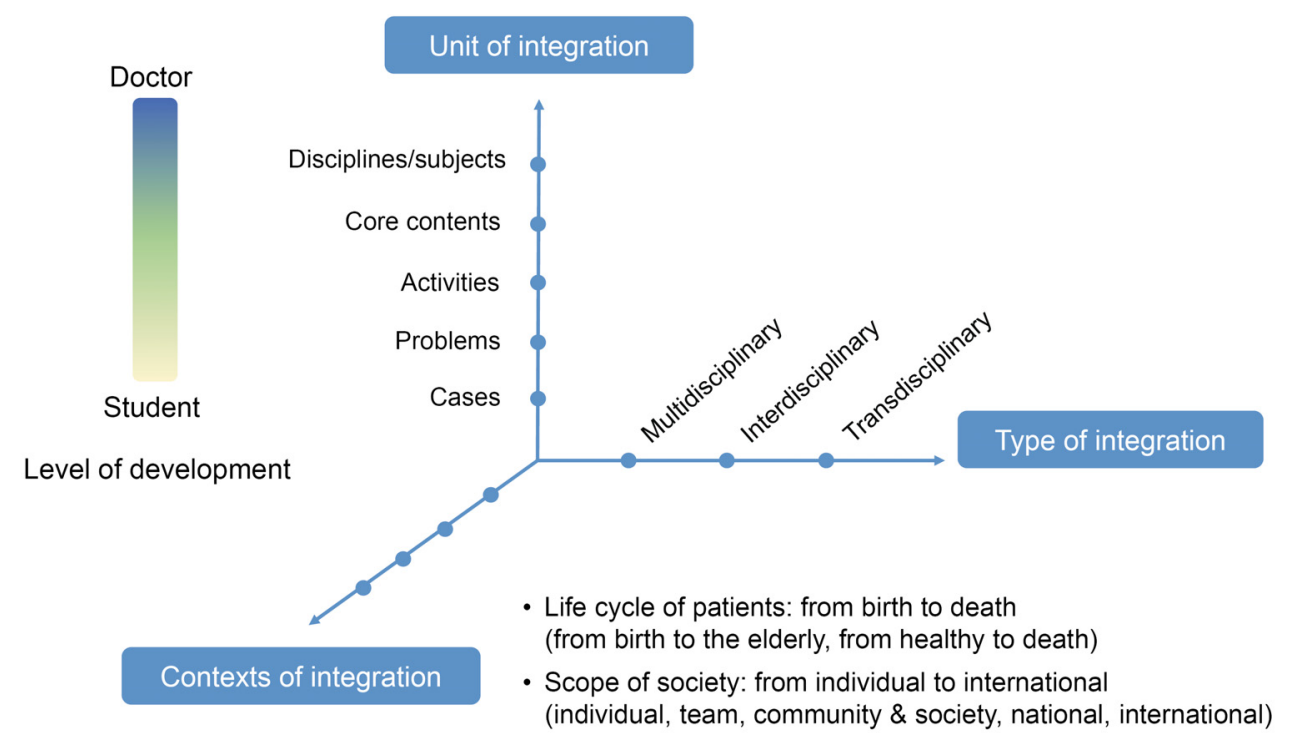

Figure 2. Factors involved in curriculum development for the medical humanities. 
먼저 IMHC 모형에서 제안하는 통합의 단위는 크게 5 가지이다. 통합의 단위를 추출하기 위하여 Kim et al. (2012)의 연구와 함께 Wolfinger \& Stockard (1997)의 교육과정 통합을 위한 접근방법과 Kim (2011)이 제안한 STEAM 교육을 위한 큐빅모형을 검토한 후, 의료인문학에서 활용 가능한 5 개의 통합단위를 도출하였으며, 이는 통합의 규모에 따라 사례 및 현상, 문제, 체험활동, 핵심내용, 학문 및 교과로 나뉜다. 즉 통합 의료인문학 교육과정 개발을 위해서는 사회적 현상이나 각종 사례 기반의 통합, 문제기반의 통합, 학생들이 직접 참여하여 경험할 활동을 기반으로 한 통합, 학습목표 즉 학습할 핵심내용에 근거한 통합, 그리고 학문 혹은 교과 중심의 통합이 가능하다.

$\mathrm{IMHC}$ 모형에서 제안하는 통합 시 고려해야 할 두 번째 요소는 이러한 통합단위를 어느 정도로 통합시켜 줄 수 있는지를 나타내는 통합의 유형이다. 이는 Kim et al. (2012)의 연구에서와 마찬가지로 Drake (1993)가 제시한 다학문적(multidisciplinary), 간학문적 (interdisciplinary), 탈학문적(transdisciplinary) 통합으로 정의하였 다. Drake (1993)에 따르면 다학문적 통합은 한 학문을 기준으로 다른 학문을 배치하여 상호작용을 하게 하는 방식을 의미하며, 간학 문적 통합은 여러 학문의 개념, 방법, 절차가 문제의 해결을 위해 자유롭게 활용되는 방식을 의미한다. 그리고 탈학문적 통합은 학문 의 경계를 없애고 해당 목표를 달성하기 위하여 새로운 학문영역으 로 각 내용과 요소들을 통합하는 방식을 의미한다. 따라서 교과나 수업수준에서 어떤 통합유형을 따르는지에 따라 교육내용과 방법의 구성이 달라질 수 있다.

$\mathrm{IMHC}$ 모형에서 제안하는 통합 시 고려해야 할 세 번째 요소는 통합의 맥락이다. STEAM 모형에서는 통합의 맥락을 개인적 맥락, 지역-사회적 맥락, 세계적 맥락의 수준으로 정의한 바 있다. 본 연구 진은 의료인문학 교육과정에서 다루어지는 교과내용의 맥락을 크게 2가지로 정의하고, 각 맥락별 수준을 정의하였다. 첫 번째 맥락은 환자의 생애주기에 따른 맥락으로 출생 이후 성장발달 단계에 따른 접근 혹은 건강한 상태에서 죽음에 이르는 질환의 진행 단계에 따른 접근 등 시간의 흐름에 따른 맥락이다. 두 번째 맥락은 개인이 속한 사회조직의 확장 정도에 따른 것으로 개인, 팀, 공동체와 사회, 국가; 그리고 세계로 정의하였다. 이러한 맥락은 의료인문학의 여러 내용 이 의학과 체계적으로 통합될 수 있도록 함과 동시에 교육내용의 제시 순서 및 교육과정의 순서(sequence)를 결정하는 데도 도움을
줄 수 있다.

\section{4. 학생의 발달 정도}

마지막으로 본 연구진은 교육내용의 제시 순서 및 교육과정의 순서를 결정하는 데 도움을 주는 요소로 학생의 발달 정도(level of development)를 고려해야 한다고 제안한다. 이는 의료인문학 교육과정이 '의사되기(doctoring)' 과정이라는 이해를 바탕으로 한 명의 학생이 사회에서 요구하는 교양인으로 성장하고, 이후 좋은 의사로 성장해가는 일련의 의사되기 발달과정을 경험한다는 전제를 바탕으로 제시되었다. 또한 통합된 내용이나 교육과정이 학생의 발 달과정에서 어느 정도 수준으로 이루어져야 하는지를 고려함으로써 동일 내용을 어떻게 심화시킬 것인지 혹은 어떤 맥락에서 제시할 것인지 등에 대한 의사결정이 수월해질 것이며, 제시될 내용이나 목표가 어느 수준으로 구성되어야 하는지를 결정하는 데도 도움이 될 것이다. 본 연구에서는 학생의 발달 정도를 역량기반 성과바탕 교육과정에서 주로 논의되는 수준(level)과 동일하게 정의하였다. 특히 영남대학교 의과대학에서 도입한 4 개 수준 개념으로 정의하고 졸업 후 전공의 교육에서 요구되는 수준인 전문가 수준을 제외한 3 개 수준으로 발달수준을 정의하였다. 이는 초보자, 중급자, 숙련자 이다(Table 4).

\section{IMHC 모형을 활용한 통합 의료인문학 교육과정 예시}

형성적 연구절차에 따라 잠정적 이론 모형인 $\mathrm{IMHC}$ 모형의 적용 가능성을 검토하기 위하여 통합 형태로 개발된 대표적 사례들을 분석하였다. 그 사례는 계명대학교 의과대학(이후 계명의대)에서 운영되고 있는 '의료윤리(medical ethics), 영남대학교 의과대학(이 후 영남의대)에서 운영되고 있는 '의학개론(introduction to medicine)'과 '의학과 인문학의 만남(introduction to medical humanities),' 그리고 '의학과 문화(medicine \& culture)' 교육과정이었 다. 이 교육과정들은 전체 교육과정 통합 전 교과단위로 개발된 통합 교육과정들이다.

\section{1. 의료윤리 교육과정 중 '치료중단과 사전의향' 사례 예}

먼저 계명의대에서 운영되고 있는 의료윤리 교육과정 중 '치료중 단과 사전의향 사례에 대하여 $\mathrm{IMHC}$ 모형을 적용해 본 결과는

Table 4. Level of students

\begin{tabular}{llll}
\hline & \multicolumn{1}{c}{ Beginner } & \multicolumn{1}{c}{ Intermediate } & \multicolumn{1}{c}{ Advanced } \\
\hline Knowledge & $\begin{array}{l}\text { Recalling \& understanding concepts \& } \\
\text { principles }\end{array}$ & Applying concepts \& principles & Applying knowledge \& problem solving \\
Skill & Understanding/observation & Copying/simulation & Simulation/performance with supervisor \\
Attitude & Acceptance & Response & Value/internalization \\
\hline
\end{tabular}




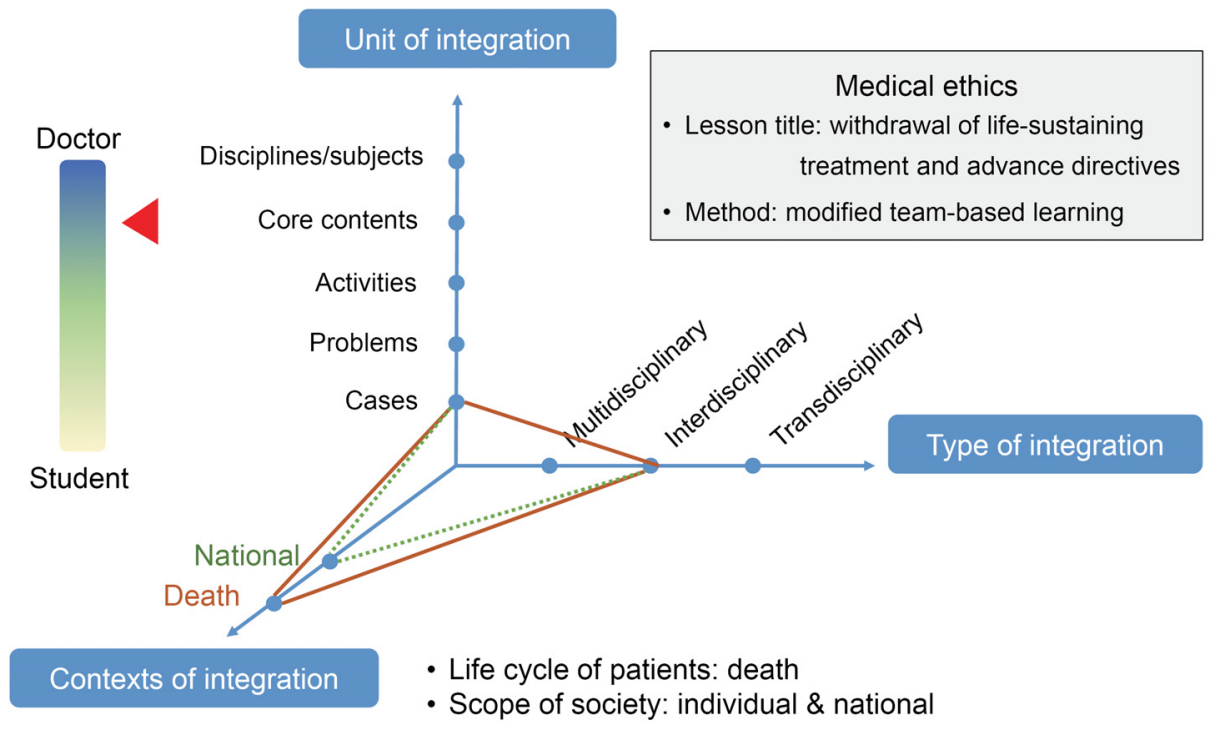

Figure 3. Analysis of medical ethics by theoretical model of integrated medical humanities curriculum.

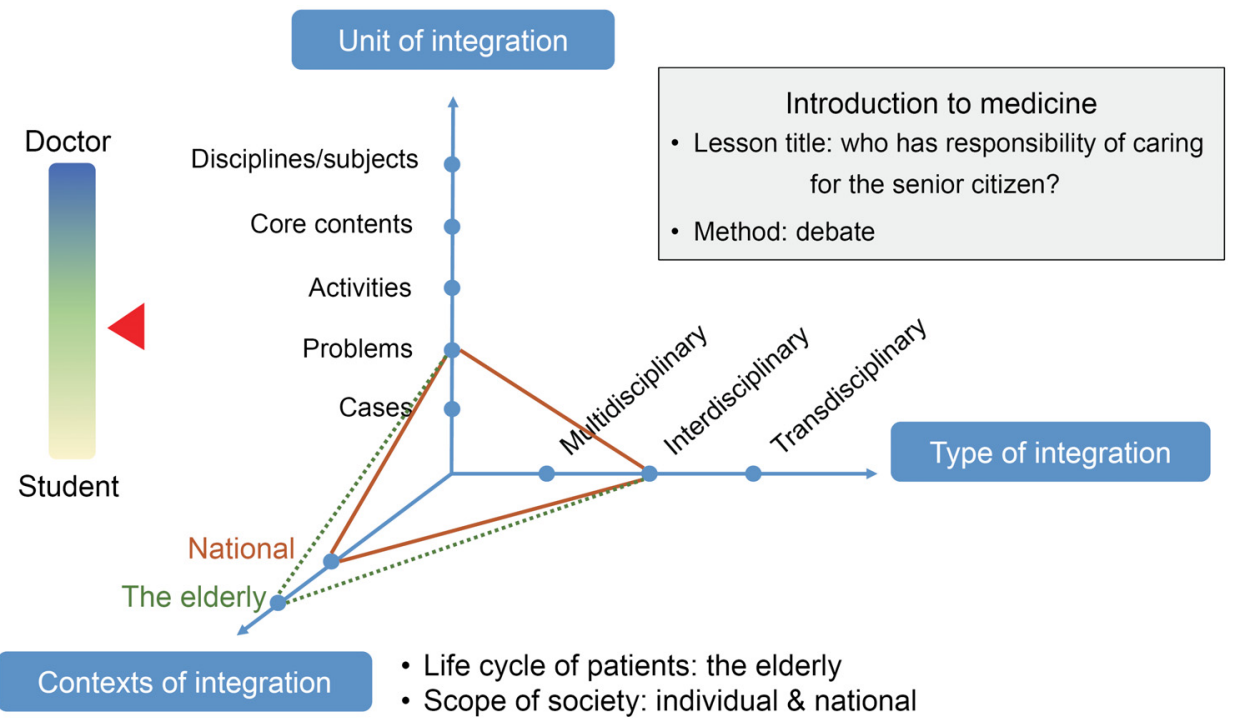

Figure 4. Analysis of introduction to medicine by theoretical model of integrated medical humanities curriculum.

Figure 3과 같다. 의료윤리 교육과정은 프로페셔널리즘, 의사소통, 윤리 및 도덕적 추론, 그리고 문제해결 역량을 갖추는데 필요한 교육과정이며, '윤리와 의료윤리의 이해,' '의료에 대한 이해,' '의사 로서의 책무성' 등에 대한 내용을 포함하고 있다. 이 교육과정은 의학과 2학년에 개설되어 있으며, 단위시간당 2시간 동안의 팀 기반 학습형태로 운영되고 있고 개설 학기 내에 총 5 개 의료윤리 사례에 대한 토론수업으로 진행된다. 이 중 1개 사례가 '치료중단과 사전의 향이다. 이 수업에 본 모델을 적용해본 결과 해당 교과의 통합단위는 사례이며, 통합유형은 간학문적 통합방식으로 구성되었고, 통합의 맥락은 환자의 생애주기 중 ‘죽음'과 관련된 맥락을 개인적 수준과
국가적 수준 모두에서 고려하도록 구성하고 있다. 이 교과는 의과대 학생의 발달단계 중 중상위 발달단계, 즉 고학년 수준에 적합한 교과로 분석되었다. 이 수업은 의학과 2학년에서 3학년 수업으로 개선될 예정이다.

\section{2. 의학개론 교육과정 예}

다음으로 영남의대에서 운영되고 있는 '의학개론' 교육과정에 대한 IMHC 모형을 적용해본 결과는 Figure 4와 같다. 의학개론 교육과정은 프로페셔널리즘, 의사소통, 문제해결, 그리고 사회와 공동체 기반의 의료와 관련된 역량을 갖추는데 필요한 교육과정이 
며, '의료에 대한 이해,' '인접학문에 대한 이해,' '의사소통기술,' '의사의 사회적 책임감' 등에 대한 내용을 포함하고 있다. 이 교육과 정은 의예과 2학년에 개설되어 있으며, 학기당 8-12개의 문제에 대한 디베이트 수업으로 운영되고 있다. 이 중 1 개의 문제가 '노인부 양의 책임은 누구에게 있는가? 이다. 이 수업에 본 모델을 적용해본 결과 해당 교과의 통합단위는 문제 중심이며, 통합유형은 간학문적 통합방식이다. 통합의 맥락은 환자의 생애주기 중 '노년기’와 관련된 맥락을 개인적 수준과 국가적 수준 모두에서 고려하도록 구성하고 있다. 이 교과는 의과대학생의 발달단계 중 중간 정도의 발달단계에 적합한 교과로 분석되었다.

\section{3. 의학과 인문학의 만남 교육과정 예}

다음으로 영남의대에서 운영되고 있는 '의학과 인문학의 만남' 교육과정에 대한 $\mathrm{IMHC}$ 모형을 적용해본 결과는 Figure 5와 같다. 의학과 인문학의 만남 교육과정은 자기개발과 사회와 공동체 기반의 의료와 관련된 역량을 갖추는데 필요한 교육과정이며, 의료인문학 입문을 위한 통합 교육과정으로 개발되었다. 주요 교육내용은 '인간 과 고통에 대한 이해,' '자신에 대한 분석과 개발' '의료와 사회의 관계에 대한 이해' 등이며 의예과 2학년에 개설되어 있다. 16 차시의 강의와 함께 프로젝트 기반학습이 진행되고 있으며, ' good work project'에 기반을 두어 본 모델을 적용해본 결과는 다음과 같다.

\section{Unit of integration}

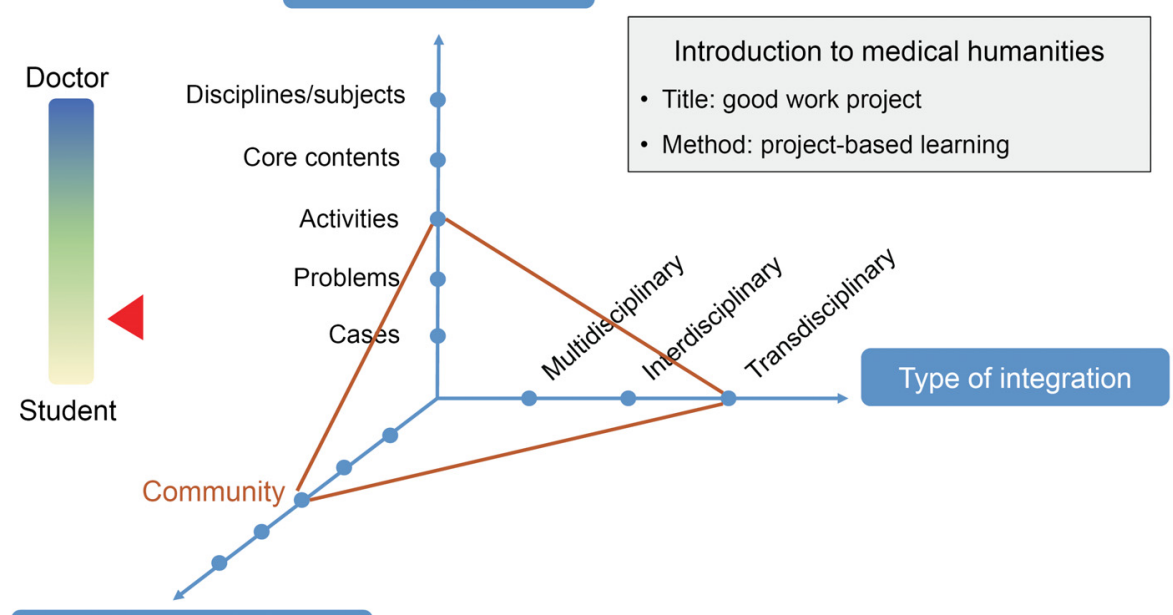

\section{Contexts of integration - Scope of society: individual \& community}

Figure 5. Analysis of introduction to the medical humanities by theoretical model of integrated medical humanities curriculum.

\section{Unit of integration}

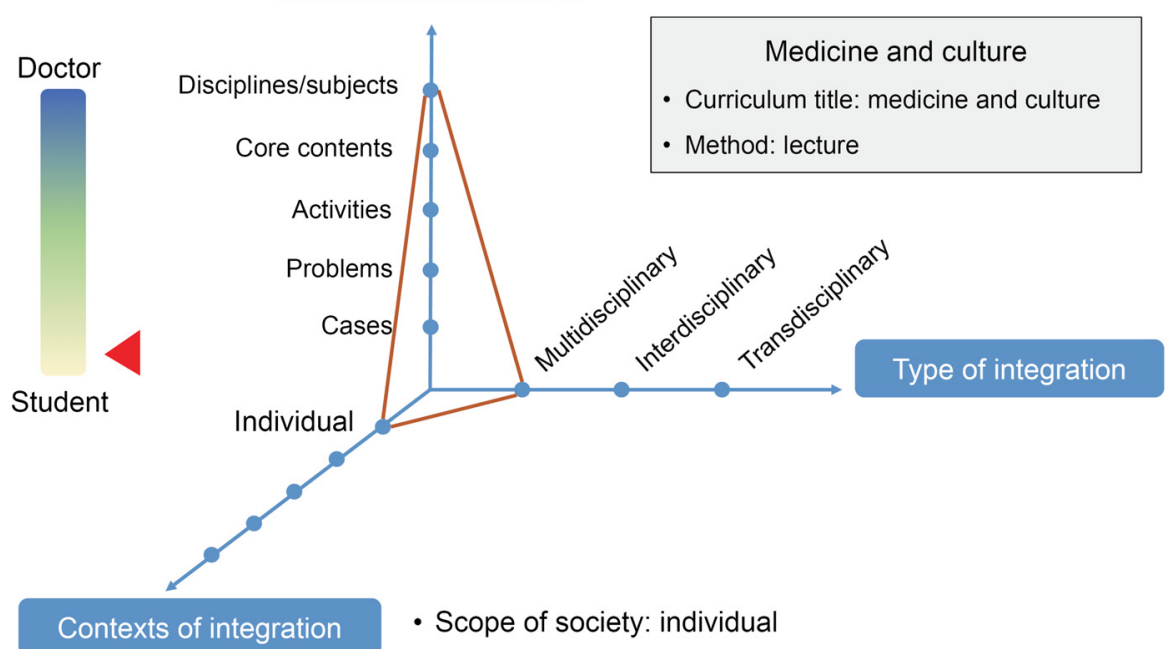

Figure 6. Analysis of introduction to medicine \& culture by theoretical model of integrated medical humanities curriculum. 
해당 교과의 통합단위는 활동이며, 통합유형은 탈학문적 통합방식 이다. 통합의 맥락은 개인적 수준과 공동체 수준에서 고려하도록 구성하고 있다. 이 교과는 의과대학생의 발달단계 중, 중 이하의 발달 단계에 적합한 교과로 분석되었다.

\section{4. 의학과 문화 교육과정 예}

마지막으로 영남의대에서 운영되고 있는 '의학과 문화' 교육과정 에 대한 분석결과는 Figure 6과 같다. 의학과 문화 교육과정은 자기 개발 역량과 관련된 교육과정이며, '인간에 대한 이해,' '인문학에 대한 이해,' '종교와 문화에 대한 이해' 등을 주요내용으로 한다. 현재 의학과 1학년에 개설되어 있으며, 문화를 대표하는 예술관련 주제들을 해당 영역의 전문가가 특강하는 형태로 운영되고 있다. 이 교과목에 대하여 본 모델을 적용해 본 결과는 다음과 같다. 해당 교과의 통합단위는 학문/교과이며, 통합 유형은 다학문적 통합방식 이다. 통합의 맥락은 개인적 수준이며 의과대학생의 발달단계 중 초기 발달단계에 적합한 교과로 분석되었다. 즉 교양교과로 적합하 다. 이 교육과정은 6 년제 교육과정으로의 개선 시 의학 전 기초교육 과정 시기에 배치될 예정이다.

\section{결 론}

의료인문학 교육과정은 일반 학생이 의사로 성장할 수 있도록 사회화하는 과정이라는 점에서 교양교과로서의 인문학과 사회과학 을 넘어 의학적 맥락과 문제들을 충실히 내포하는 통합 교육과정으 로의 개발과 개선이 요구된다. 본 연구는 통합 의료인문학 교육과정 개발을 위한 이론모형을 제안함으로써 교과 및 교육과정 개선 실무 자들을 위한 가이드라인을 제공하고, 교육학적 근거에 입각한 교육 과정의 개발과 개선을 돕기 위한 목적으로 진행되었다. 통합교육과 정 모형 중 하나인 융합인재교육(STEAM)을 위한 이론적 모형(Kim et al., 2012)의 틀에 기반을 두어 통합의료인문학 교육과정 이론모형 을 도출하였다. 해당 모형에 대한 숙의와 검증, 그리고 개선과정을 거쳐 최종 이론모형을 확정하였으며, 이 모형은 크게 대학의 교육성 과 즉 의료인문학 관련 핵심역량과 핵심 교육내용, 그리고 통합의 요소영역으로 구성되며, 추가 고려요인으로 학생의 발달수준을 도 출하였다.

첫 번째 영역인 핵심역량은 통합 의료인문학 교육과정의 종료 시 학생들이 획득해야 하는 역량으로서 최종 성과를 의미한다. 학문 중심으로 이루어져 온 인문학 혹은 사회과학이 의료인문학이라는 통합적 영역으로 수용될 때는 반드시 해당 대학이나 교육기관이 추구하는 인재상과 그 인재가 갖추어야 할 역량을 달성할 수 있는 형태로 조정 혹은 개선되어야 할 것이다. 의료인문학의 필요성과 의료인문학의 교육의 성과는 바로 핵심역량에 대한 규명과 이해에서 시작된다는 것이 본 연구진의 첫 번째 합의사항이었다.
두 번째 영역인 핵심내용은 통합의 대상이자 반드시 교육되어야 할 전체 교육과정 내용과 목표를 의미한다. 교육과정 개선에서 가장 먼저 요구되는 것은 의료인문학 교육과정의 표준이 될 목표에 대한 것이었다. 2007년도에 한국의과대학-의학전문대학원협회에서 제 시한 목표들은 이미 8년 이상 경과된 것이며, 해당 목표들에 대한 구체적인 검증이나 적절성에 대한 검토는 거의 이루어지지 않았다. 본 연구에서는 통합 의료인문학 교육과정을 '의사되기' 과정으로 보고, 교육과정 목표들과 함께 프로페셔널리즘의 주요 요소들 (Cruess et al., 2009)을 비교하고, 용어의 적절성과 추가적으로 교육 해야 하는 교육내용을 점검하였다. 그 결과 의료인문학 과정 전체가 직업전문성을 포함하고 있으며, 포괄적인 관점을 적용하여 그 하위 요인들의 내용을 반영하였을 경우 ‘의사의 직업전문성'은 교육과정 목표의 하위영역으로는 적절치 못한 것으로 판정되었다. 이에 교육 과정 목표를 '전문가로서의 품행이해'로 수정하고 그 하위영역에 '의사로서의 직업적 책무'를 추가하였다. 추후 이러한 목표의 수정이 적합한지에 대한 전문가 의견조사와 이에 따른 질적 양적 연구가 추가적으로 요구된다.

세 번째 영역인 통합요소는 실제 교육과정 통합을 위해 반드시 고려되어야 하는 요소들을 규정한 것으로서 본 연구에서는 STEAM 모형의 틀에 기반을 두어 단위, 유형, 그리고 맥락요소를 주요 하위요 소로 수용하였다. 각각의 요소들의 특성은 다시 숙의과정을 거쳐 의료인문학에 적합한 수준들로 재도출하였고, 해당 요소에 학생의 발달단계요소를 추가함으로써 전체 4 개의 통합요소들을 고려하여 교육과정을 개발 혹은 개선해야 함을 제안하는 바이다. 통합의 단위 는 사례, 문제, 활동, 내용, 그리고 교과나 학문의 5 가지 수준으로, 통합의 유형은 다학문적, 간학문적, 탈학문적 수준으로 정의하였으 며, 의료인문학의 가장 중요한 고려요소로 통합의 맥락을 환자의 생애주기와 개인이 속한 사회조직의 확장 정도 2 가지로 정의하였다. 또한 학생의 발달 정도에 따라 시기성과를 반영할 수 있는 초보자, 중급자, 숙련자의 3 단계 분류방식을 도출하였다.

이러한 통합요소들을 고려함으로써 수업이나 교과 혹은 교육과 정 전체의 내용, 방법, 시기를 명료하게 정의하여 평가할 수 있다. $\mathrm{IMHC}$ 모형을 최근 개발되고 운영되고 있는 4 개의 의료인문학 통합 교육과정에 적용해 본 결과 이 이론적 모형의 적용이 해당 교과의 특성과 시기 결정에 도움을 줄 수 있는 것으로 확인되었으며, 수업수 준, 과정수준, 교육과정수준에서 모두 적용 가능한 것으로 확인되어 이를 통합 의료인문학 교육과정의 잠정적 이론모형으로 확정하였다. 본 연구자들은 이 모형이 다음의 측면에서 활용될 수 있을 것으로 기대한다. 첫째, 현재 진행되고 있는 의료인문학 교육과정의 개발 및 개선과정에서 학문중심의 교과들을 물리적 형태로 나열하는 표면 적 수준의 통합방식에서 벗어나 교과의 구조와 기능을 고려한 진정 한 통합의 시도를 촉진하도록 안내할 수 있을 것이다. 둘째, 개발 및 개선에 참여하는 교수설계자나 교과내용 전문가의 의사소통을 
돕고 실제 의사결정과정에서 활용 가능한 가이드라인으로 활용될 수 있을 것이다. 셋째, 통합의 목표, 내용, 방법, 수준이 적절한지에 대한 평가의 틀로서 활용될 수 있을 것이며, 이를 기반으로 교육과정 개발과 개선에 참여할 여러 유관 학문 전문가들 간의 합의를 도출하 는 데 있어 그 수월성과 용이성을 증진시킬 수 있을 것이다.

본 연구의 한계와 추후 연구제안은 다음과 같다. 먼저 본 연구는 의료인문학 통합 교육과정 구성에 대한 이론 모형을 제안하는 것이 다. 따라서 통합 시 고려해야 하는 요소들에 관한 이론모형 연구에 이어 교수설계 모형과 관련된 추후 연구가 요구되며 교수방법, 평가 방법 등 운영과 관련된 구체적인 가이드라인이 개발되어야 할 것이 다. 아울러 추후 해당 모형의 적합성과 타당성을 검증하기 위하여 2 개 의과대학 소속 연구진들의 숙의를 거친 형성연구 수준을 넘어 전문가 평가와 실제 사례분석이 추가적으로 이루어져야 할 것이다. 또한 의료인문학 교육에서 다루어야 하는 핵심역량과 이를 교육하기 위한 교육 내용들에 대한 검토를 확대하고, 각 역량별 혹은 내용에 맞는 학습경험과 교육방법의 적절성 등을 제안하는 연구가 진행될 필요가 있으며, 본 연구의 후속연구로 구체적인 적용방안과 적용경 험에 대한 연구들이 활발히 이루어지기를 기대한다.

\section{감사의 글}

본 연구는 2012년도 계명대학교 비사연구기금으로 이루어졌다.

\section{REFERENCES}

Baek, Y. S., Park, H. J., Kim, Y. M., Noh, S. G., Park, J. Y., Lee, J. Y., ... Han, H. S. (2012). A study on the action plans for STEAM education. Seoul: Korea Foundation for the Advancement of Science and Creativity.

Baik, S. H. (2010). The way of making a good doctor. Korean J Med Educ, 22(4), 249-256.

Cruess, R. L., Cruess, S. R., \& Steinert, Y. (2009). Teaching medical professionalism. New York: Cambridge University press.

Drake, S. M. (1993). Planning integrated curriculum. Alexandria (VA): Association for supervision and curriculum development.

Han, J. J. (2013). The development of outcome-based curriculum in medical schools outside Korea. Korean Med Educ Rev, 15(1), 19-24.

Jeon, W. T., Kim, S. H., \& Oh, S. M. (2010). Medical humanities. Seoul: Young Doctors.

Jeon, W. T., \& Yang, E. B. (2003). Future of medical humanities and medical education. Seoul: Yonsei University Press.

Kang, H. S., \& Ju, D. B. (2004). Curriculum \& educational evaluation. Seoul: Hakjisa.

Kim, J. S. (2011). A cubic model for STEAM education. Korea Cit Index, 11(2), 124-139.

Kim, S. W., Chung, Y. L., Woo, A. J., \& Lee, H. J. (2012). Development of a theoretical model for STEAM education. Korea J Assoc Sci Educ, 32(2), 388-401.

Kwon, S. O. (2005). Conception of medical humanities and it's role in medical education. Korean J Med Educ, 17(3), 217-223.

Reigeluth, C. M. (1999). Instructional-design theories and models, volume II. New York: Lawrence Associates Inc.

The Korean Council of Medical College Deans. (2007). Educational goals of medical college. Seoul: The Korean Council of Medical College Deans.

Wolfinger, D. M., \& Stockard, J. S. Jr. (1997). Elementary method: An integrated curriculum. New York: Longman. 